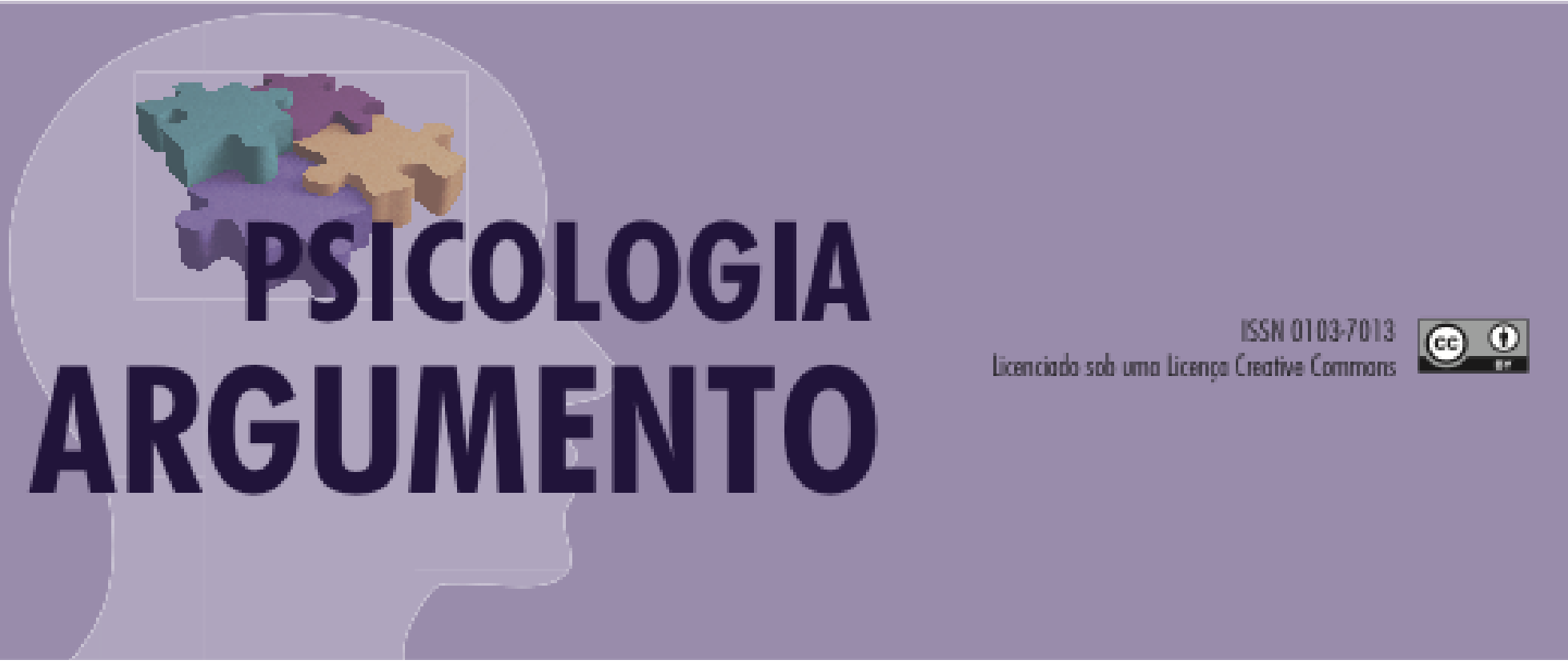

doi: http://dx.doi.org/10.7213/psicolargum.38.99.AO05

\title{
Reflexões sobre um treinamento em Entrevista Motivacional em estudantes da área da saúde
}

Reflections on Motivational Interview training for students in the health field

Reflexiones sobre la capacitación en Entrevista Motivacionale para estudiantes de salud

Fernanda Pasquoto Souza

Professora titular da Universidade Luterana do Brasil

email: fepgs@terra.com.br ORCID: http://orcid.org/0000-0002-3230-4688

\section{Marina Heinen}

Mestranda - Psicologia Clínica, Pontifícia Universidade Católica do Rio Grande do Sul. email: m.marinaheinen@gmail.com ORCID: http://orcid.org/0000-0001-9362-2295

\section{Margareth da Silva Oliveira}

Docente do programa de pós graduação em psicologia da PUCRS Email:

marga@pucrs.br ORCID: http://orcid.org/0000-0002-6490-5170

\section{Resumo}

O uso da Entrevista Motivacional (EM) pode auxiliar o paciente na ativação das suas metas, configurando-se como um modo de comunicação eficaz para mudanças no estilo de vida. Entretanto, é necessário que o profissional esteja capacitado para a aplicação dos princípios da EM, a fim de ser uma prática efetiva. O objetivo deste estudo foi refletir sobre a importância do uso da EM no campo da saúde. Para isso, avaliou-se o nível de conhecimento em EM e a aplicação das habilidades fundamentais da EM em uma amostra de 120 estudantes da área da saúde, após 
um treinamento. Verificou-se o grau de Confiança e de Importância em utilizar essas habilidades na prática profissional dos participantes. Encontrou-se que quanto maior o grau de confiança em EM, maior é o percentual de perguntas consistentes com os fundamentos da EM e maior chance de acertos na EM. Os achados mostram a relevância do treinamento de Entrevista Motivacional nos cursos da área da saúde, pois potencializam a confiança dos alunos ao trabalharem com os pacientes sobre mudanças de comportamento e qualificam a formação do profissional da área da saúde.

Palavras-Chave: Entrevista Motivacional; Programa de Treinamento; Comportamentos Relacionados com a Saúde; Comportamentos de Risco à Saúde

\begin{abstract}
The use of Motivational Interviewing (MI) can help the patient to activate his goals, configuring himself as an effective way to communicate for changes in lifestyle. However, it is necessary that the professional is trained to apply the principles of MI in order to be an effective practice. The aim of this study was to reflect on the importance of using MI in the health field. To do this, use the level of knowledge in MI and an application of basic MI skills in a sample of 120 students in the health field, after training in MI. The degree of confidence and importance in the use of these skills in the participants' professional practice was verified. It was found that the greater the degree of confidence in MI, the greater the percentage of questions consistent with the fundamentals of MI and the greater the probability of success in MI. The results show the relevance of motivational interview training in health care courses, as they improve students' confidence when working with patients in behavioral changes and qualify the training of health professionals.
\end{abstract}

Keywords: Motivational Interview; Training program; Health-Related Behaviors; Health Risk Behaviors

\title{
Resumen
}

El uso de la Entrevista Motivacional (EM) puede ayudar al paciente a activar sus objetivos, configurándose como una forma efectiva de comunicación para los cambios en el estilo de vida. Sin embargo, es necesario que el profesional esté capacitado para aplicar los principios de la EM, a fin de ser una práctica efectiva. El objetivo de este estudio fue reflexionar sobre la importancia del uso de la EM en el campo de la salud. Para hacer esto, use el nivel de conocimiento en EM y una aplicación de habilidades básicas en EM en una muestra de 120 estudiantes en el campo de la salud después de la capacitación en EM. Se verificó el grado de confianza e importancia en el uso de estas habilidades en la práctica profesional de los participantes. Se encontró que cuanto mayor es el grado de confianza en la EM, mayor es el porcentaje de preguntas consistentes con los fundamentos de la EM y la mayor probabilidad de éxito en la EM. Los resultados muestran la relevancia de la capacitación de la entrevista motivacional en los cursos de atención médica, ya que mejoran la confianza de los estudiantes cuando trabajan con pacientes en cambios de comportamiento y califican la capacitación de los profesionales de la salud.

Palabras clave: Entrevista Motivacional; Programa de entrenamiento; Comportamientos relacionados con la salud; Comportamientos de riesgo para la salud

\section{Introdução}

Os profissionais das diferentes áreas da saúde podem levar um tempo significativo da consulta tentando convencer o paciente do seu comportamento potencialmente danoso à saúde. Entretanto, as pesquisas têm demonstrado que um comportamento não pode ser 
modificado com sucesso se o paciente não estabelecer as próprias metas e internalizar a necessidade de mudança. Devido ao desafio dos profissionais da saúde em ajudar o paciente a alcançar e manter a mudança de um comportamento tem-se havido um considerável interesse em estudos sobre a eficácia e utilização da Entrevista Motivacional (EM) no âmbito da saúde (Segatto, Andreoni, Souza, Diehl \& Pinsky, 2011; Brandinelli, Gonçalves \& Fonseca, 2013).

Inicialmente desenvolvida para pacientes com histórico de abuso e dependência de substâncias psicoativas, a EM tem sido adaptada e utilizada em diferentes contextos da área da saúde (Miller \& Rollnick, 2013; Rollnick, Miller \& Butler, 2009; Miller \& Moyers, 2017). A EM é uma abordagem baseada em evidências que visa o aumento da motivação do paciente para mudar o estilo de vida disfuncional e aumentar o comprometimento com a mudança (Miller \& Rollnick, 2013). A EM é uma intervenção focada na dificuldade do paciente, podendo ser realizada em um único momento ou durante o tratamento, tornando-se útil nos serviços de atenção primária e viável de ser utilizada por uma gama de profissionais em diferentes serviços (Miller \& Moyers, 2017; Kaltman, WinklerPrins, Serrano \& Talisman, 2015). Na entrevista, o profissional se relaciona de forma empática e não confrontativa com o paciente. Através de um estilo colaborativo, o profissional conduz uma conversa, respeitando a autonomia do paciente em resolver a ambivalência sobre as mudanças específicas de comportamento em prol de saúde (Miller \& Rollnick, 2013).

Alguns comportamentos podem prejudicar o andamento da entrevista, não sendo consistentes com os princípios da EM, por exemplo: o uso de perguntas fechadas, aconselhamentos, julgamentos e confrontação. Por isso, Miller e Rollnick (2013) enfatizam as habilidades básicas da EM. São elas: (1) Pergunta Aberta que permite uma gama de possibilidades de respostas por parte do paciente; (2) Postura empática do profissional em relação à perspectiva do paciente; (3) Afirmação que consiste em dizer algo que estimule o paciente a manter um determinado comportamento durante a consulta; (4) Reflexão que envolve capturar ou devolver em forma de resposta uma declaração do paciente e (5) Resumo, reunindo o que foi dito pelo paciente e permitindo a transição para o próximo tópico da conversa.

Estudos apontam o uso da EM como uma ferramenta importante no tratamento de pacientes portadores de diferentes doenças, como asma e insuficiência cardíaca. Os 
resultados dessas pesquisas apontam que, após a intervenção com EM, houve aumento na adesão do plano de tratamento e no auto-cuidado desses pacientes (Román-Rodrigues et al., 2017; Guimarães et al., 2019). Entretanto, uma revisão sistemática apontou que embora a EM tenha sido eficaz em 75\% dos estudos analisados, há a necessidade de novos estudos sobre a eficácia da prática com a EM em diferentes contextos (Brandinelli et al., 2013).

A Entrevista Motivacional se espalhou para além da psicologia clínica, como nas áreas da saúde, serviço social, educação e reabilitação, impactando a vida de muitos pacientes (Miller \& Moyers, 2017). Nesse sentido, estudos demonstram que uma ampla variedade de profissionais tem sido treinada com sucesso para utilizar efetivamente a EM na prática clínica (Schoo, Lawn, Rudnik \& Litt, 2015; Chéret et al., 2018; Faustino-Silva et al., 2019).

Apesar das evidências sobre as vantagens de utilizar a EM com pacientes no âmbito da saúde, a EM permanece subutilizada pelos diferentes profissionais da saúde no Brasil, isso pode ocorrer devido a não familiaridade ou insegurança em utilizar essa modalidade de entrevista (Segatto et al., 2011). Nesse sentido, estudos recentes sugerem que os fundamentos da EM sejam inseridos nas aulas de formação e sirvam como orientação para as diferentes profissões relacionadas à saúde (Schoo et al., 2015; Fortune, Breckon, Norris, Eva \& Frater, 2019). Tendo em vista essa carência e para que haja maior eficácia e disseminação da EM, torna-se necessário investimentos em programas de treinamento para estudantes da área da saúde.

Nos Estados Unidos, observa-se que diversas universidades oferecem tanto na grade curricular ou como atividade extraclasse um treinamento em EM para estudantes dos cursos da área da saúde, como enfermagem, fisioterapia, nutrição, psicologia, odontologia e medicina (Coumbe-Lilley \& Weidner, 2014). A Universidade de Hong Kong através do projeto intitulado "Motivational interviewing in undergraduate clinical education: An opportunity for a new interprofessional learning experience" (Gao et al., 2014) oferece, desde 2013, um treinamento em EM para estudantes dos cursos de odontologia, medicina e enfermagem.

Uma revisão sistemática da literatura indicou que os treinamentos em EM podem facilitar o uso proficiente das estratégias por diferentes estudantes e profissionais da área da saúde (De Souza, Da Silva, Boff, Trindade \& Oliveira, 2012). Uma pesquisa apontou 
que quando as habilidades da EM foram incorporadas nas disciplinas os estudantes relataram maior capacidade e confiança ao utilizar os conhecimentos dessa abordagem para os atendimentos (Kaltman et al., 2015; Schoo et al., 2015). Entretanto, há pesquisas que relatam a falta de preparo dos estudantes em auxiliar os pacientes na mudança de comportamentos (Hall, Staiger, Simpson, Best \& Lubman, 2016).

Nessa perspectiva, o objetivo deste estudo foi refletir sobre a importância do uso da EM no campo da saúde. Para isso, avaliou-se o nível de conhecimento em EM e a aplicação das habilidades fundamentais da EM por estudantes da área da saúde, após um treinamento. Além disso, objetiva-se comparar o uso dessas habilidades entre os cursos. Verificou-se, também, o grau de confiança e da importância em utilizar essas habilidades básicas na prática diária dos participantes.

\section{Método}

Trata-se de um estudo quasi-experimental, explicativo, descritivo e transversal. Serão apresentadas as avaliações após o treinamento. Esse estudo faz parte de um Edital CAPES (Coordenação de Aperfeiçoamento de Pessoal de Nível Superior) chamado Ensino Saúde, que envolve um projeto de integração entre Universidades e Políticas de Saúde, com o objetivo de promover ações voltadas para o ensino, pesquisa, formação e qualificação profissional em temas da saúde.

\section{Participantes}

Participaram da pesquisa 120 estudantes do último ano de graduação dos cursos de Medicina, Fisioterapia, Odontologia, Psicologia, Enfermagem e Nutrição de uma universidade privada do sul do Brasil, escolhidos por conveniência. Foram incluídos no estudo homens e mulheres que estavam regularmente inscritos em um dos cursos citados. Adotou-se como critério de exclusão já ter realizado algum treinamento formal em EM.

\section{Instrumentos}

Ficha de Dados Sócio-demográficos: Construiu-se um questionário descritivo para coleta de dados sócio demográficos (sexo, idade, estado civil, etnia). Além disso, investigou qual o curso de graduação e se já realizou algum treinamento em EM. 
Régua de Importância e de Confiança: Foi desenvolvida por Miller e Rollnick (2002) com o objetivo de avaliar a importância e confiança em usar a EM. Para avaliar a importância, o participante deve responder a pergunta: "Quão importante você diria que é aprender sobre Entrevista Motivacional?” e em uma escala em forma de régua o participante deve marcar 0 , nada importante, até 10, extremamente importante. Já para avaliar a confiança, o participante deve responder a pergunta: "Quão confiante você diria que está, se decidir usar a Entrevista Motivacional em seus atendimentos, de que poderia ter sucesso?", e deve marcar em uma régua de 0 a 10 , sendo 0 nada confiante e 10 extremamente confiante.

Exercício de Entrevista Dialogada: Para identificar as habilidades básicas da EM utilizou-se uma vinheta retirada do livro de Rollnick, Miller e Butler (2009). A vinheta refere-se a um diálogo entre profissional e paciente. O profissional informa sobre o resultado elevado do exame de colesterol do paciente e segue com a técnica da Entrevista Motivacional. Para maior clareza, apresenta-se um recorte da vinheta: Paciente: "Mas eu estou preocupado com o efeito que parar de fumar possa ter na minha dieta" - Profissional: "Então o senhor está preocupado que parar de fumar possa aumentar o seu peso e ser ruim". Após o treinamento, solicitou-se que o participante identificasse, a partir da vinheta, um exemplo para cada um dos itens a seguir: pergunta aberta, afirmação, resumo, escuta reflexiva e resistência. As respostas foram pontuadas como acerto ou erro.

Helpful Responses Questionnaire (HRQ): O Questionário de Resposta Útil, foi desenvolvido por Miller, Hedrick e Orlofsky em 1991. Esse instrumento contém seis hipotéticas declarações de pacientes e solicita-se que o participante escreva "o que diria em seguida", em resposta a cada declaração. Um exemplo de declaração é "Eu realmente me sinto péssimo. Ontem à noite eu me embebedei e nem mesmo lembro do que fiz. Esta manhã descobri que a tela da televisão está quebrada e eu penso que provavelmente fiz isso, mas minha esposa nem mesmo está falando comigo. Eu não penso que eu sou um alcoólatra, você sabe, porque eu posso ficar semanas sem beber. Mas isso tem que mudar". O objetivo é avaliar até que ponto o aluno utilizou pergunta aberta, afirmação, reflexão, resumo, foi empático, aconselhou ou julgou cada declaração. Este questionário é uma alternativa quando a observação individual não é possível, assim como quando os indivíduos estão sendo avaliados em um treinamento. Avaliou-se os comportamentos do participante em relação ao percentual de perguntas abertas ou fechadas e se o participante 
foi consistente com fundamentos da EM (empatia, perguntas abertas, reflexão, afirmação, resumo). Essa avaliação pautou-se em uma parte dos indicadores de habilidades em EM conforme indicado pelo instrumento Motivational Interviewing Skill Code (MISC) (Miller, Moyers, Ernst \& Amrhein, 2008).

Procedimentos de coleta de dados

Após aprovação do projeto de pesquisa pelo Comitê de Ética, efetuou-se contatos com os professores dos diferentes cursos da área da saúde com o objetivo de apresentar a pesquisa e solicitar a colaboração para a divulgação do treinamento em sala de aula. Os alunos foram informados pelos professores dos objetivos e organização do treinamento. Os alunos interessados preencheram a ficha de dados sócio demográficos e, após leitura e esclarecimento de possíveis dúvidas, assinaram o Termo de Consentimento Livre e Esclarecido (TCLE). Os participantes receberam o treinamento e após o término da intervenção houve a avaliação do grupo.

Toda a coleta de dados foi realizada por membros do grupo de pesquisa, treinados para este fim específico. Já os coordenadores do treinamento possuem treinamento avançado em EM no Squaretop (Albuquerque, USA). É importante ressaltar que os treinamentos foram oferecidos pelo mesmo profissional, que é formado em Psicologia.

\section{Treinamento em Entrevista Motivacional}

Realizou-se um treinamento de 20 horas, dividido em dois sábados com horário das $8 \mathrm{~h}$ às $18 \mathrm{~h}$, para habilitar os estudantes na utilização das estratégias básicas da EM, as quais se mostram eficazes com os diferentes tipos de problemas mais comumente abordados na área da saúde. O treinamento enfatizou a compreensão teórica e o desenvolvimento de habilidades em EM. A concentração deu-se em uma das habilidades específicas da EM que é provocar a "conversa sobre mudança", etapa que antecede a ação para a mudança.

O treinamento visou permitir que o estudante conheça sobre os fatores envolvidos na mudança de comportamento e sobre o papel da motivação e ambivalência, compreenda o espírito, os princípios e os métodos da Entrevista Motivacional, reconheça e acompanhe a resistência do paciente, utilize as habilidades da escuta reflexiva para promover a mudança, adquira experiência, através da demonstração e da prática de exercícios e 
aprenda e pratique as habilidades de comunicação que são úteis no desenvolvimento de relações de confiança e de colaboração com os pacientes e que ajudam a aumentar o seu interesse em realizar mudanças. Espera-se que a partir do treinamento os alunos sejam capazes de reconhecer e utilizar os componentes da Entrevista Motivacional, utilizando técnicas para provocar uma conversa focada na mudança.

O treinamento foi apresentado em um formato experiencial, com orientações práticas para temas como uso de habilidades de comunicação empática, uso de reflexão simples e avançada para trabalhar com a resistência e a ambivalência, desenvolver discrepância, escutar e provocar a "conversa sobre mudança". O uso dos princípios da motivação, métodos e estratégias foram apresentados através de uma combinação de palestras, estudos de casos, vídeos e exercícios em duplas e em pequenos grupos. $\mathrm{O}$ treinamento ocorreu em um espaço cedido pela universidade que os alunos estudavam.

\section{Procedimentos de análise de dados}

Todas as análises foram realizadas usando o pacote estatístico SPSS (Statistical Package for the Social Sciences) versão 23.0. As estatísticas descritivas foram calculadas para todas as variáveis, utilizando média e desvio padrão (DP) para variáveis contínuas e frequências e percentagens para as variáveis categóricas. As variáveis ordinais foram tratadas como variáveis categóricas. Foram utilizadas as fórmulas do percentual de acertos na vinheta, calculado sob a fórmula: $\left(\mathrm{n}^{\mathrm{o}}\right.$ de acertos nos cinco comportamentos/total de comportamentos) x 100.

Os percentuais de perguntas fechadas e abertas para as seis questões do Questionário de Resposta Útil, foram calculados da seguinte maneira: ( $n^{\circ}$ de questões onde foi usada a pergunta aberta ou fechada/total de questões) x 100. Também avaliouse o percentual consistente com EM através da fórmula: $\left(n^{0}\right.$ de questões onde foram utilizados comportamentos compatíveis com EM/total de questões) x 100. O mesmo raciocínio foi empregado para o cálculo do percentual inconsistente com EM. Para testar as diferenças entre os cursos a análise de Variância One-way complementada pelo teste de Tukey (two-tailed) foi realizada para variáveis contínuas e qui-quadrado para variáveis categóricas. O coeficiente de correlação de Pearson foi empregado para testar as correlações. A análise de Covariância (ANCOVA) com ajuste por Bonferroni foi aplicada 
para controlar a influência de fatores confundidores na relação entre os cursos e os desfechos. Valores de $\mathrm{p}<0,05$ foram considerados significativos.

\section{Procedimentos éticos}

Os procedimentos foram realizados respeitando os preceitos éticos, diretrizes e normas estabelecidas na Resolução n. ${ }^{\circ}$ 466/2012 do Conselho Nacional de Saúde - CNS - (Brasil, 2012), que regulamenta as pesquisas envolvendo seres humanos. Informou-se sobre a participação voluntária, sigilo dos dados e benefícios do estudo. Esse estudo teve parecer favorável pelo Comitê de Ética em Pesquisa (CAAE 18995613.2.0000.5336).

\section{Resultados}

A amostra foi composta por 120 alunos de diferentes cursos da área da saúde. A média de idade da amostra foi de 24,7 anos $( \pm 6,7)$, com predominância do sexo feminino $(88,3 \%)$. As estatísticas descritivas por curso estão apresentadas na Tabela 1. Foi possível observar que os alunos do curso de Nutrição apresentaram média de idade significativamente superior à dos alunos da Medicina e Enfermagem.

\begin{tabular}{|c|c|c|c|c|c|c|c|}
\hline Variáveis & $\begin{array}{c}\text { Psicologia } \\
n=20 \\
16,7 \%\end{array}$ & $\begin{array}{c}\text { Enfermagem } \\
n=20 \\
16,7 \%\end{array}$ & $\begin{array}{c}\text { Nutrição } \\
n=20 \\
16,7 \%\end{array}$ & $\begin{array}{c}\text { Odontologia } \\
n=20 \\
16,7 \%\end{array}$ & $\begin{array}{c}\text { Medicina } \\
\mathrm{n}=20 \\
16,7 \%\end{array}$ & $\begin{array}{c}\text { Fisioterapia } \\
n=20 \\
16,7 \%\end{array}$ & $\mathrm{p}$ \\
\hline $\begin{array}{l}\text { Idade (anos) - média } \pm \text { DP } \\
\text { Sexo }-\mathrm{n}(\%)\end{array}$ & $24,7 \pm 7,4^{\mathrm{ab}}$ & $23,6 \pm 4,1^{\mathrm{a}}$ & $29,7 \pm 11,4^{b}$ & $24,0 \pm 3,5^{\mathrm{ab}}$ & $22,7 \pm 2,7^{\mathrm{a}}$ & $23,9 \pm 5,0^{\mathrm{ab}}$ & 0,012 \\
\hline $\begin{array}{l}\text { Masculino } \\
\text { Feminino }\end{array}$ & $\begin{array}{c}3(15) \\
17(85)\end{array}$ & $\begin{array}{c}0(0) \\
20(100)\end{array}$ & $\begin{array}{c}0(0) \\
20(100)\end{array}$ & $\begin{array}{c}5(25) \\
15(75)\end{array}$ & $\begin{array}{c}4(20) \\
16(80)\end{array}$ & $\begin{array}{c}2(10) \\
18(90)\end{array}$ & 0,066 \\
\hline
\end{tabular}

$\mathrm{DP}=$ Desvio Padrão;

a,b Letras iguais não diferem pelo teste de Tukey a 5\% de significância

Na Tabela 2 estão apresentados os resultados dos instrumentos aplicados posteriormente ao treinamento. Após o ajuste pela idade, permaneceram significativas as diferenças entre os cursos quanto ao percentual de perguntas abertas ser consistente com a EM e nas Réguas de Importância e Confiança. Em relação ao percentual de Perguntas Abertas e ter um comportamento consistente com a EM, os alunos dos cursos de Odontologia e Fisioterapia apresentaram menor proporção quando comparados aos da Enfermagem.

Quando verificada a Régua de Importância, os alunos do curso de Nutrição consideram mais importante usar a EM do que as alunas do curso Enfermagem. Quanto à Régua de Confiança, os alunos de Enfermagem e Fisioterapia têm maior confiança em 
utilizar a EM quando comparados aos alunos de Odontologia. Apenas entre esses cursos houve diferenças significativas.

Para o entendimento da influência das características demográficas nos desfechos, a idade foi avaliada em três faixas etárias ( $<25$ anos, de 25 a 30 anos e mais de 30 anos). Houve diferença significativa entre as faixas etárias quanto ao percentual de acertos na vinheta $(\mathrm{p}=0,034)$, sendo que o percentual de acertos aumenta com o avanço da idade,

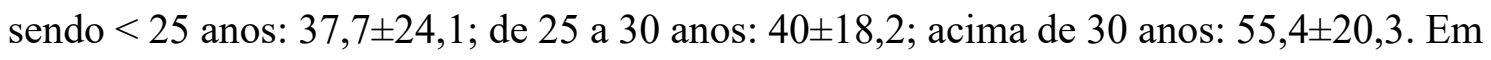
relação ao gênero, as diferenças não foram significativas ( $\mathrm{p}>0,05)$.

Tabela 2.

Comparação dos desfechos entre os cursos

\begin{tabular}{|c|c|c|c|c|c|c|c|c|c|}
\hline Variáveis & $\begin{array}{l}\text { Amostra total } \\
\quad(n=120)\end{array}$ & $\begin{array}{c}\text { Psicologia } \\
\mathrm{n}=20 \\
16,7 \%\end{array}$ & $\begin{array}{c}\text { Enfermagem } \\
\mathrm{n}=20 \\
16,7 \%\end{array}$ & $\begin{array}{c}\text { Nutrição } \\
n=20 \\
16,7 \%\end{array}$ & $\begin{array}{c}\text { Odontologia } \\
n=20 \\
16,7 \%\end{array}$ & $\begin{array}{c}\text { Medicina } \\
\mathrm{n}=20 \\
16,7 \%\end{array}$ & $\begin{array}{c}\text { Fisioterapi } \\
\mathrm{a} \\
\mathrm{n}=20 \\
16,7 \%\end{array}$ & $\mathrm{p}$ & pajustado* \\
\hline \multicolumn{10}{|l|}{ Vinheta (número de acertos) } \\
\hline Pergunta Aberta & $77(64,2 \%)$ & $15(75 \%)$ & $16(80 \%)$ & $15(75 \%)$ & $13(65 \%)$ & $10(50 \%)$ & $8(40 \%)$ & 0,050 & - \\
\hline Afirmação & $37(30,8 \%)$ & $6(30 \%)$ & $9(45 \%)$ & $7(35 \%)$ & $4(20 \%)$ & $9(45 \%)$ & $2(10 \%)$ & 0,105 & - \\
\hline Resumo & $41(34,2 \%)$ & $4(20 \%)$ & $9(45 \%)$ & $9(45 \%)$ & $8(40 \%)$ & $5(25 \%)$ & $6(30 \%)$ & 0,407 & - \\
\hline Escuta Reflexiva & $41(34,2 \%)$ & $8(40 \%)$ & $4(20 \%)$ & $11(55 \%)$ & $6(30 \%)$ & $10(50 \%)$ & $2(10 \%)$ & 0,019 & - \\
\hline Resistência & $45(37,5 \%)$ & $6(30 \%)$ & $7(35 \%)$ & $8(40 \%)$ & $10(50 \%)$ & $5(25 \%)$ & $9(45 \%)$ & 0,588 & - \\
\hline Percentual de acertos total & $40,2 \pm 22,8^{\mathrm{ab}}$ & $39,0 \pm 23,8^{\mathrm{ab}}$ & $45,0 \pm 24,2^{\mathrm{ab}}$ & $50,0 \pm 26,4^{b}$ & $41,0 \pm 20^{\mathrm{ab}}$ & $39,0 \pm 22^{\mathrm{ab}}$ & $27,0 \pm 14,9^{\mathrm{a}}$ & 0,040 & 0,096 \\
\hline \multicolumn{10}{|l|}{ Questionário de resposta útil } \\
\hline Perguntas Abertas (\%) & $23,3 \pm 24,4^{\mathrm{ab}}$ & $25,8 \pm 21,9^{\mathrm{ab}}$ & $35,0 \pm 21,6^{\mathrm{b}}$ & $30,8 \pm 28,2^{\mathrm{ab}}$ & $13,3 \pm 25,1^{\mathrm{a}}$ & $\begin{array}{c}22,5 \pm 22,5^{\mathrm{a}} \\
\mathrm{b}\end{array}$ & $12,5 \pm 20,1^{\mathrm{a}}$ & 0,013 & 0,020 \\
\hline Perguntas Fechadas (\%) & $19,7 \pm 23,1$ & $23,3 \pm 26,7$ & $20,0 \pm 21,4$ & $16,7 \pm 20,2$ & $25,8 \pm 27,8$ & $20,8 \pm 25,3$ & $11,7 \pm 14,4$ & 0,455 & 0,475 \\
\hline Consistente com EM & $35,7 \pm 31,7^{\mathrm{ab}}$ & $41,7 \pm 29,4^{\mathrm{ab}}$ & $48,3 \pm 24,7^{b}$ & $38,3 \pm 29,2^{\mathrm{ab}}$ & $18,3 \pm 25,3^{\mathrm{a}}$ & $46,7 \pm 42,4^{b}$ & $20,8 \pm 25,3^{\mathrm{a}}$ & 0,004 & 0,002 \\
\hline Régua Importância & $7,55 \pm 2,49^{\mathrm{ab}}$ & $7,85 \pm 2,43^{\mathrm{ab}}$ & $6,05 \pm 3,30^{\mathrm{a}}$ & $9,00 \pm 1,49^{b}$ & $7,60 \pm 1,57^{\mathrm{ab}}$ & $\underset{\mathrm{b}}{7,30 \pm 2,00^{\mathrm{a}}}$ & $7,50 \pm 2,91^{\mathrm{ab}}$ & 0,010 & 0,010 \\
\hline Régua Confiança & $6,48 \pm 3,12^{\mathrm{ab}}$ & $5,95 \pm 3,20^{\mathrm{ab}}$ & $7,90 \pm 2,90^{\mathrm{b}}$ & $6,85 \pm 2,48^{\mathrm{ab}}$ & $4,20 \pm 2,73^{\mathrm{a}}$ & $\underset{\mathrm{b}}{6,25 \pm 3,02^{\mathrm{a}}}$ & $7,70 \pm 3,16^{b}$ & 0,001 & 0,001 \\
\hline
\end{tabular}

Nota. EM=Entrevista Motivacional;

${ }^{\mathrm{a}, \mathrm{b}}$ Letras iguais não diferem pelo teste de Tukey a 5\% de significância;

* ajustado pela idade por Análise de Covariância (ANCOVA)

Na relação entre os instrumentos, houve associação significativa entre a Régua de Importância com o percentual de acertos total na vinheta $(r=0,229 ; p=0,012)$. Desta forma, quanto mais o aluno julga importante a EM, maior o percentual de acertos total na vinheta, como pode ser visto na Figura 1. 


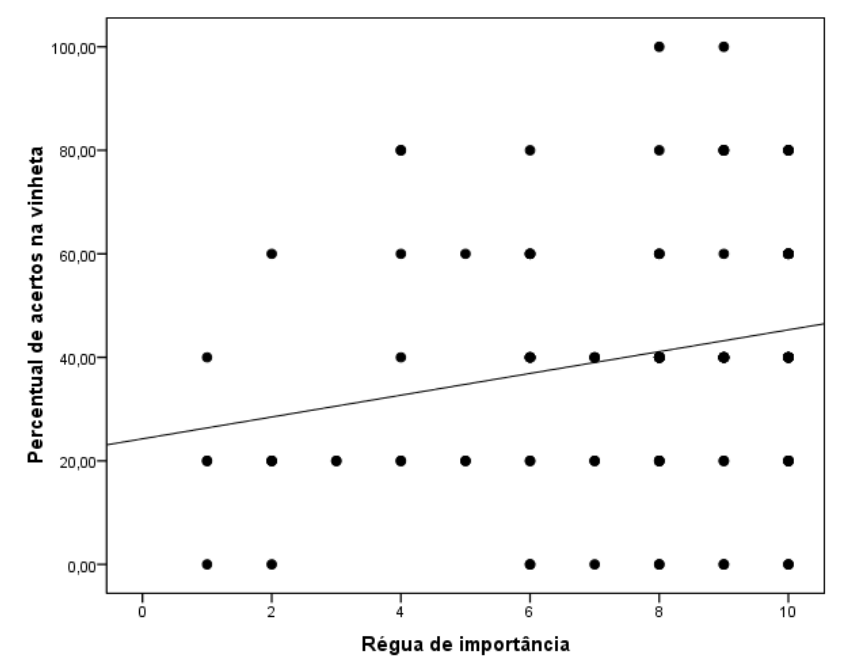

Figura 1

Associação entre a Régua de Importância e o percentual de acertos na vinheta.

Com a Régua de Confiança, houve associação significativa com o percentual de perguntas compatíveis com a EM $(r=0,272 ; p=0,003)$. Sendo assim, quanto mais o aluno confia na EM, maior o percentual de perguntas Consistentes com a EM no Questionário de Resposta Útil, como visualizado na Figura 2.

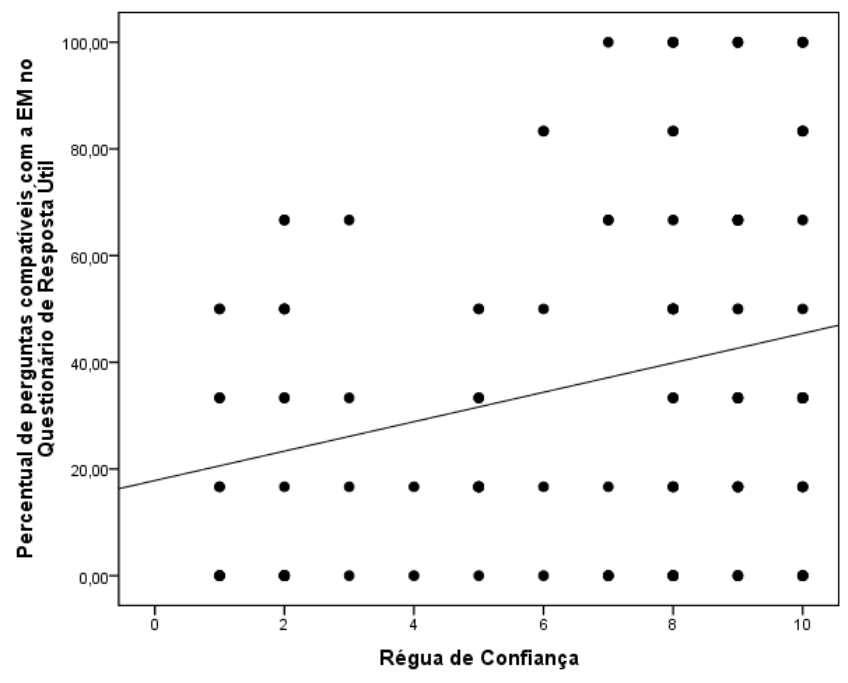

Figura 2

Associação entre a Régua de Confiança e o percentual de perguntas Consistentes com a Entrevista Motivacional no Questionário de Resposta Útil. 
Por fim, também houve associação entre o percentual de perguntas consistentes com a EM no Questionário de Resposta Útil e o percentual de acertos na EM ( $\mathrm{r}=0,185$; $\mathrm{p}=0,043$ ). Portanto, quanto maior o percentual de perguntas consistentes com a EM, maior o percentual de acertos na EM.

\section{Discussão}

O objetivo deste estudo foi refletir sobre a importância do uso da EM no campo da saúde, além de avaliar o nível de conhecimento das habilidades fundamentais da Entrevista Motivacional dos alunos de diferentes cursos de graduação da área da saúde. Objetivouse também comparar possíveis diferenças entre os cursos. Além disso, avaliou-se o quanto os participantes sentem-se confiantes e acreditam ser importante utilizar essas habilidades na prática clínica. Essas avaliações foram realizadas apenas após os participantes serem submetidos a um treinamento em EM. Vale ressaltar que não houve avaliação pré treinamento.

No presente estudo, houve associação significativa entre os instrumentos do protocolo. Os resultados demonstram que os alunos que acertaram mais na vinheta também acertaram mais no Questionário de Resposta Útil, o que demonstra a consistência nos nossos achados. Os estudantes da amostra acreditam ser importante ter as habilidades básicas da EM.

Interessantemente, os alunos da Enfermagem não consideraram tão importante o uso da EM, mas sentem-se confiantes em relação às estratégias básicas, se fossem utilizá-las. Ter mais confiança pode ser congruente com o fato dos alunos do curso de Enfermagem apresentarem maior domínio sobre o uso de Perguntas Abertas e ser consistente com a EM do que os demais cursos. Esse resultado pode estar relacionado à prática do profissional da enfermagem estar atrelada a tarefas mais operacionais. Esse resultado pode ser reforçado com os achados de um estudo atual, o qual apontou que embora a EM apresente evidências de efetividade, a utilização das técnicas da EM não é integrada de maneira padronizada no trabalho dos enfermeiros (Hontangas et al., 2019). Frente a isso, percebe-se como fundamental o investimento em treinamentos durante a formação para qualificar o ensino e a prática dos atendimentos posteriormente. Assim, treinamentos em EM no âmbito acadêmico podem auxiliar os profissionais na compreensão da importância 
do uso da técnica na prática, como apresenta o estudo de Hontangas et al (2019), em que os estudantes submetidos a um treinamento em EM perceberam como uma oportunidade para a aquisição de habilidades e reflexão sobre o uso eficaz da EM com os pacientes.

Os estudantes de Fisioterapia apresentaram menor média tanto no nível de acertos quanto na utilização de perguntas abertas. Isso pode ser justificado pelos poucos estudos ou investimento na formação em EM dos profissionais dessa área. Essa hipótese é corroborada pelos resultados de uma revisão sistemática da literatura que evidenciou que as profissões mais estudadas em EM são medicina, enfermagem, nutrição, assistência social e psicólogos (De Souza et al., 2012).

Já os alunos de Odontologia apresentaram baixo nível de perguntas abertas e baixa confiança e consistência com o uso da EM. Entretanto, diferentemente dos achados nesta pesquisa, os estudos atuais demonstram que o uso da EM tem sido efetivo no campo da Odontologia para mudanças de comportamento dos pacientes, como a aderência ao tratamento (Faustino-Silva et al., 2019; Silva, Silva \& Cascaes, 2019).

Os participantes do curso de Psicologia não apresentaram destaque nas variáveis avaliadas. Entretanto apresentaram pontuações médias de acertos, perguntas abertas e em ser consistente com EM. Uma provável explicação para esse resultado diz respeito à possibilidade dos estudantes de Psicologia já terem algum contato com a EM, visto que esse tema é comum de ser abordado nessa área da saúde em comparação com os demais cursos. A comparação entre o conhecimento prévio e após o treinamento não foi objetivo deste estudo. Por isso, frente a essa hipótese, sugere-se que futuras pesquisas sejam realizadas para discutir essa temática e comparar o conhecimento dos estudantes e entre os cursos antes e depois de um treinamento em EM.

Segundo a Health Foundation (2011) e os resultados da revisão sistemática e metanálise de Rubak, Sandbæk, Lauritzen e Christensen (2005) não há evidências se um determinado tipo de profissional da saúde é mais eficaz na utilização da EM do que outro, como por exemplo, médicos versus enfermeiros versus psicólogos, ou se é possível prever quem pode fazer uso com mais sucesso dessa abordagem. Apesar de se tratar de uma metodologia prática e objetiva, o profissional deve estar capacitado para utilizar a EM, a fim de ser uma estratégia efetiva (Figlie \& Guimarães, 2014). Da mesma forma, segundo 
esses autores, não há provas suficientes para identificar quais características demográficas possam prever se uma determinada graduação será mais eficaz quando utilizar a EM. No nosso estudo foi possível observar que o percentual de acertos na vinheta não permaneceu significativo após o ajuste pela idade. Provavelmente, isso ocorreu porque os alunos mais velhos têm maior percentual de acertos na vinheta.

A utilização da EM em diferentes abordagens de tratamento e práticas clínicas foi evidenciada em uma revisão sistemática da literatura, que apontou um aumento na procura de profissionais da área da saúde por formação em EM (De Souza et al., 2012). Estudos internacionais que objetivaram avaliar programas baseados nas habilidades relacionadas à EM relataram que a aplicação de treinamentos específicos para EM aumentou a compreensão e a capacidade dos alunos, de diferentes áreas da saúde, de realizar entrevista baseada nos princípios da EM com pacientes (Schoo et al., 2015; Bailey, Currington, Brown, Hegener \& Espel, 2017; Fortune et al., 2019; Chéret et al., 2018). Um estudo brasileiro recente apontou que um treinamento em EM aumentou a confiança e as habilidades dos estudantes da área da saúde em utilizar os princípios da EM com pacientes, melhorando o desempenho no atendimento durante a academia e a vida profissional (De Souza, Meyer \& Oliveira, 2019). Frente a isso, os resultados do atual estudo demonstram a importância de estudar mais profundamente, assim como disponibilizar as habilidades básicas da EM aos estudantes da área da saúde no Brasil.

Vale ressaltar que diversos cadernos de atenção básica enfatizam o tratamento baseado na Entrevista Motivacional para tabagistas, por exemplo. Entretanto, não há ênfase nas graduações da área da saúde em como o profissional pode auxiliar um paciente a lidar com a ambivalência frente à mudança de um comportamento. Assim, torna-se necessário investir e defender a formação voltada para atuação na saúde pública. A literatura atual enfatiza que o treinamento em EM deve ser incorporado no currículo de diferentes áreas da saúde para preparar os alunos na prática profissional (Fortune et al., 2019). Uma pesquisa informou que programas educacionais para desenvolver habilidades da EM em estudantes da área da saúde pode resultar em uma aprendizagem inovadora e maior prontidão para praticar os princípios da EM (Schoo et al., 2015). Esse trabalho e os resultados obtidos trazem à tona a possibilidade de implantar disciplinas eletivas no currículo acadêmico de universidades, com o intuito de disseminar e treinar os estudantes 
da área da saúde sobre os princípios da EM. O formato em aulas ou seminários pode diminuir a dificuldade ou resistência dos atendimentos e aumentar a eficácia da intervenção.

Ter conhecimento em Entrevista Motivacional pode promover a maior utilização dessa técnica, o que contribuirá para a prática clínica. A importância do treinamento em EM pode ser reforçada visto que é aplicável em uma grande variedade de problemas que envolve o cuidado da saúde, desde o incentivo ao uso regular da medicação até um comportamento de saúde específico. O uso da EM é especialmente interessante para os profissionais da área da saúde que atendem pessoas que necessitam aumentar a adesão ao plano de tratamento, mudar hábitos inadequados ou comportamentos não saudáveis (Miller \& Rose, 2009). Segundo Rollnick, Butler, Kinnersley, Gregory e Mash (2010) a EM tem demonstrado promover a mudança de comportamento em vários cenários da saúde, melhorar a relação profissional-paciente e a eficiência da consulta.

\section{Considerações Finais}

Os resultados desse estudo devem ser considerados no contexto de certas limitações metodológicas. Primeiro, quantificar as respostas dos participantes não teve como objetivo avaliar a qualidade das respostas, mas simplesmente contá-las. Além disso, o MISC foi desenvolvido como uma ferramenta para avaliar a competência na EM de terapeutas e não foi especificamente avaliada como um instrumento para medir o desempenho nas habilidades básicas da EM dos estudantes de graduação. Ademais, o estudo buscou avaliar, como uma prova de conhecimentos, as habilidades e conhecimentos da EM após o treinamento, não tendo o objetivo de avaliar e apresentar os dados antes e após o treinamento. Por fim, esse estudo usou uma amostra de estudantes de apenas uma universidade e os resultados não são passíveis de generalização. Apesar dessas limitações, o presente estudo contribui para o conhecimento sobre a relevância de avaliar o nível de conhecimento dos alunos de diferentes cursos na área da saúde, sobre as habilidades básicas da EM. 
Esse estudo oportunizou algumas compreensões sobre o conhecimento das habilidades básicas da EM com uma amostra de estudantes de diferentes cursos da área da saúde que permitem identificar direções para futuras pesquisas. A "conversa sobre mudança" feita pelo paciente é evocada e reforçada pelas ações do profissional da saúde durante o atendimento com o objetivo de aumentar a probabilidade de que a mudança de comportamento alcançada seja mantida. Nesse sentido, torna-se necessário que os alunos aprendam as habilidades para comunicar-se com os pacientes de forma eficaz na abordagem dos comportamentos relacionados à saúde.

Nossos achados mostram a relevância de introduzir um treinamento de EM nos cursos da área da saúde, pois, independente do curso, encontrou-se que quanto maior o grau de confiança em EM, maior é o percentual de perguntas consistentes com os fundamentos da EM e maior chance de acertos na EM. Esse estudo sugere que a inclusão da EM no currículo formal ou extraclasse para estudantes das diferentes áreas da saúde pode oportunizar o desenvolvimento do conhecimento e da aplicação do espírito e das habilidades fundamentais da Entrevista Motivacional em suas carreiras. Além disso, implementar fundamentos da EM na graduação engloba ir ao encontro de ações de qualificação na formação do profisssional da área da saúde.

As aulas focadas em Entrevista Motivacional poderão melhorar a confiança dos alunos ao falar com pacientes sobre mudanças de comportamento em prol da saúde. Futuras pesquisas são necessárias para examinar a eficácia de um treinamento em Entrevista Motivacional para uma maior amostra de estudantes de diferentes áreas da saúde no Brasil, visando a expansão do conhecimento e da confiança na capacidade em implementar a mudança de comportamentos com os pacientes no âmbito da saúde.

\section{Referências}

Bailey, L., Curington, R., Brown, B., Hegener, M., \& Espel, M. (2017). Motivational interviewing education: Creation and assessment of a learning module implemented among advanced pharmacy practice students. Currents in Pharmacy Teaching and Learning, 9(5), p. 786-793. https://doi.org/10.1016/j.cptl.2017.06.007 
Brandinelli, L. P., Gonçalves, H. A., \& Fonseca, R. P. (2013). A entrevista motivacional e sua aplicabilidade em diferentes contextos: uma revisão sistemática. Revista da Sociedade de Psicologia do Rio Grande do Sul, 13(1), p. 26-34. Recuperado de: http://www.sprgs.org.br/diaphora/ojs/index.php/diaphora/article/view/82/82

Brasil. (2012). Ministério da Saúde. Conselho Nacional de Saúde. Comissão Nacional de Ética em Pesquisa. Recuperado de:

http://conselho.saude.gov.br/web_comissoes/conep/index.html

Chéret, A., Durier, C., Noel, N., Bourdic, K., Legrand, C., D’Andréa, C., Hem, E., Goujard, C., Berthiaume, P., \& Consoli, S. M. (2018). Motivational interviewing training for medical students: A pilot pre-post feasibility study. Patient Education and Counseling 101(11), p. 1934-1941. https://doi.org/10.1016/j.pec.2018.06.011

Coumbe-lilley, J., \& Weidner, A. (2014). Developing Undergraduate Use of OARS: Skill Building for Senior Year Kinesiology Students. Motivational Interviewing: Training, Research, Implementation, Practice, 1(3), p. 13-19. https://doi.org/10.5195/mitrip.2014.45.

De Souza, F. P., Meyer, E., \& Oliveira, M. S. (2019). Entrevista Motivacional para Estudantes da Área da Saúde: Resultados de um Treinamento. Psicologia: Teoria e Pesquisa, 35, p. 1-7. https://doi.org/10.1590/0102.3772e3552

De Souza, F. P., Da Silva, E. M., Boff, R. M., Trindade, M. T., \& Oliveira, M. S. (2012) Características do Treinamento em Entrevista Motivacional. Aletheia, 38-39 p.186195. Recuperado de:

http://www.periodicos.ulbra.br/index.php/aletheia/article/view/3372/2510

Faustino-Silva, D. D., Colvara, B. C., Meyer, E., Hugo, F. N., Celeste, R. K., \& Hilgert, J. B. (2019). Motivational interviewing effects on caries prevention in children differ by income: A randomized cluster trial. Community Dentistry and Oral Epidemiology, 6, p. 1-8. https://doi.org/10.1111/cdoe.12488

Figlie, N. B., \& Guimarães, L. P. (2014). A Entrevista Motivacional: conversas sobre mudança. Boletim Academia Paulista de Psicologia 34(87). Recuperado de: 
http://pepsic.bvsalud.org/scielo.php?script=sci_arttext\&pid=S1415$711 X 2014000200011$

Fortune, J., Breckon, J., Norris, M., Eva, G., \& Frater, T. (2019). Motivational interviewing training for physiotherapy and occupational therapy students: Effect on confidence, knowledge and skills. Patient Education and Counseling 102(4), p. 694700. https://doi.org/10.1016/j.pec.2018.11.014

Gao, X., Bridges, S., Lo, E. C. M., Mcgrath, C., Lai, S. M. L., Chan, S. S. C., Vackova, D., \& Johnston, J. (2014). Motivational interviewing in undergraduate clinical education: An opportunity for a new interprofessional learning experience. Recuperado de: https:/tl.hku.hk/staff/teaching-development-grants/tdg-486/

Guimarães, T. M. L., Figueiredo, L. S., Velasco, N. S., Hipólito, R. L., Bandeira, G. M. S., Siqueira, M. E. B., Cavalcanti, A. C. D., \& Flores, P. V. P. (2019). A efetividade da entrevista motivacional no autocuidado de pacientes com insuficiência cardíaca: revisão sistemática. Revista Enfermagem Atual In Derme, 87 Ed. Especial. p. 1-8. Recuperado de: https://revistaenfermagematual.com.br/index.php/revista/article/view/168/70

Hall K., Staiger P. K., Simpson A., Best D., \& Lubman D. I., (2016). After 30 years of dissemination, have we achieved sustained practice change in motivational interviewing? Addiction 111(7). https://doi.org/10.1111/add.13014

Health Foundation. (2011). Research scan: Training professionals in motivational interviewing. Recuperado de:

https://www.health.org.uk/sites/default/files/TrainingProfessionalsInMotivationalInte rviewing.pdf

Hontangas, A. R., Ferrándiz, E. F., Lozoya, R. M., López, M. I. M., Guirao-Goris, S. J. A., \& Gea-Caballero, V. (2019). Evaluación de la vídeo-simulación como metodología docente para la entrevista motivacional en estudiantes de enfermería. Cultura de los Cuidados, 53, p. 239- 252. https://doi.org/10.14198/cuid.2019.53.23

Kaltman, S., WinklerPrins, V., Serrano, A., \& Talisman, N. (2015). Enhancing motivational interviewing training in a family medicine clerkship. Teaching and 
Learning in Medicine, 27(1), p. 80-4.

http://dx.doi.org/10.1080/10401334.2014.979179

Miller, W. R., Hedrick, K. E., \& Orlofsky, D. (1991) The Helpful Response

Questionnaire: a procedure for measuring therapeutic empathy. Journal of Clinical Psychology, 47(3), p. 444-448.https://doi.org/10.1002/1097-

4679(199105)47:3<444::AID-JCLP2270470320>3.0.CO;2-U

Miller, W. R., \& Rollnick, S. (2002). Motivational interviewing: Preparing people for change. 2 ed. New York: Guilford Press.

Miller, W. R., Moyers, T. B., Ernst, D., \& Amrhein, P. (2008) Manual for the Motivational Interviewing Skill Code (MISC) Version 2.1 Recuperado de: https://casaa.unm.edu/download/misc.pdf

Miller, W. R., \& Rose, G. S. (2009). Toward a theory of motivational interviewing. American Psychologist, 64(6), p. 527-537. https://doi.org/10.1037/a0016830

Miller, W. R., \& Rollnick, S. (2013). Motivational interviewing: Helping people change. 3.ed. New York: Guilford Press

Miller, W. R., \& Moyers, T. B . (2017). Motivational interviewing and the clinical science of Carl Rogers. Journal of Consulting Clinical Psychology, 85(8), p. 757766. http://dx.doi.org/10.1037/ccp0000179

Rollnick, S., Miller, W. R., \& Butler, C. (2009). Motivational interviewing in health care: helping patients change behavior. Guilford Press: New York.

Rollnick, S., Butler, C. C., Kinnersley, P., Gregory, J., \& Mash, B. (2010). Motivational interviewing. $B M J, 340$, p. 1242-1245. https://doi.org/10.1136/bmj.c1900

Román-Rodrigues, M., Ibarrola-Ruiz, L., Mora, F., Plaza, V., Sastre, J., Torrego, A., Vega, J. M., \& Sánchez-Herrero, G. (2017). Motivational interviewing for adherence: post-training attitudes and perceptions of physicians who treat asthma patients. Patient Preference and Adherence, 11, p. 811-820.

https://doi.org/10.2147/PPA.S127645 
Rubak, S., Sandbæk, A., Lauritzen, T., \& Christensen, B. (2005). Motivational interviewing: a systematic review and meta-analysis. British Journal of General Practice, 55, p. 305-312. Recuperado de:

https://www.ncbi.nlm.nih.gov/pmc/articles/PMC1463134/

Schoo, A. M., Lawn, S., Rudnik, E., \& Litt, J. C. (2015). Teaching health science students foundation motivational interviewing skills: use of motivational interviewing treatment integrity and self-reflection to approach transformative learning, BMC Medical Education, 15. https://doi.org/10.1186/s12909-015-0512-1

Segatto M. L., Andreoni S., Souza E. S. R. D., Diehl A., \& PInsky I. (2011). Brief motivational interview and educational brochure in emergency room settings for adolescents and young adults with alcohol related problems: a randomized single blind clinical trial. Revista Brasileira de Psiquiatria 33(3), p. 225-233. https://doi.org/10.1590/S1516-44462011005000004

Silva, N. R. J., Silva, A. R. R., \& Cascaes, A. M. (2019). A Entrevista Motivacional no ensino de graduação em Odontologia. Revista da ABENO, 19(3), p. 69-77. https://doi.org/10.30979/rev.abeno.v19i3.764 